\title{
REVIEW \\ Psychological variables associated with employment following spinal cord injury: a meta-analysis
}

\author{
ML Kent and DS Dorstyn
}

Objectives: Spinal cord injury (SCI) research has highlighted links between psychological variables and employment outcome; however, there remains a need to consolidate the available heterogenous data.

Methods: Meta-analytic techniques were used to examine and quantify differences in psychological functioning and employment status among adults with an acquired SCl. Fourteen observational studies ( $N=9,868$ participants) were identified from an electronic database search. Standardised mean difference scores between employed and unemployed groups were calculated using Cohen's $d$ effect sizes. Additionally, 95\% confidence intervals, fail-safe Ns, percentage overlap scores and heterogeneity statistics were used to determine the significance of $d$.

Results: Moderate to large and positive weighted effects were noted across three broad psychological constructs: affective experience or feelings $\left(d_{w}=3.16\right)$, quality of life $\left(d_{w}=1.06\right)$ and life satisfaction $\left(d_{w}=0.70\right)$. However, the most compelling non-heterogeneous finding was associated with life satisfaction, a finding that was also not subject to publication bias. Inconsistent and weak associations between employment and individual measures of vocational attitude, self-efficacy, locus of control, adjustment and personality were also noted.

Conclusion: Psychological factors and attributes are linked to employment post-SCl; however, the available data are limited in quantity. Longitudinal research is also needed to determine whether these variables can help to preserve employment over time. Spinal Cord (2014) 52, 722-728; doi:10.1038/sc.2014.92; published online 5 August 2014

\section{INTRODUCTION}

Although employment is considered to represent a key milestone in independent living and community integration following spinal cord injury (SCI), ${ }^{1,2}$ research indicates a low post-injury employment rate. $^{3-5}$ A time lag of 4.9 years (standard deviation (s.d.) =5.1) between injury onset and successful employment has also been identified, ${ }^{6}$ suggesting that any clear vocational potential in this group is not immediately realized. . $^{5}$

Researchers have now turned their attention to psychological factors and attributes as moderators of the post-SCI employment process, ${ }^{4,8,9}$ with injury and demographic characteristics considered to explain only a modest amount of variance in employment status. ${ }^{8}$ Moreover, psychological factors are potentially modifiable through clinical intervention. In their systematic review, Ottomanelli and Lind $^{4}$ reported positive role models and degree of motivation as significant contributors to post-injury employment. Qualitative research additionally indicates that engagement in a valued occupation can enhance an individual's perception of self-worth, including one's ability, competencies and capabilities. ${ }^{9,10}$ Quality of life and life satisfaction have also been associated with employment, irrespective of injury level. ${ }^{11,12}$ Additional psychological contributors include self-esteem and self-efficacy, ${ }^{13}$ personality traits, ${ }^{14}$ locus of control, ${ }^{15}$ achievement orientation, ${ }^{9}$ and work attitude or perceived importance of work. $^{14,16}$ In comparison, greater affective impairments, such as depressive symptoms, are suggested to increase the odds of being unemployed. ${ }^{17}$
However, methodological and contextual issues confound this literature. First, the psychological measures utilised in the various studies are not necessarily standardised or validated with SCI groups, hence normative data are not available. ${ }^{18}$ Second, studies have differed in relation to the interval between injury onset and employment data collection. This can affect the consistency of results, given that time since injury has been identified as a key individual difference having an impact on the dynamic psychological adjustment process following SCI. ${ }^{5}$ Finally, there is wide variability in the quantification of employment among SCI studies. Specifically, employment has been defined in terms that include the following: dichotomous categories (that is, employed vs unemployed); multiple categories (that is, fullor part-time vs student vs unemployed) and a continuous variable (that is, number of months in employment post-injury divided by the number of months available for employment). ${ }^{16}$ Although this multilayered approach incorporates a broad definition of labour market participation, by including groups such as students, volunteers, home-makers and those actively seeking work, caution needs to be taken when interpreting research that assigns different meanings to the term 'employment.' In saying this, Murphy et al. ${ }^{3}$ argue that postinjury employment achievement has validity as a marker of successful rehabilitation, due to the reliable associations that exist between it and psychological variables.

As multiple similar studies accrue in this area, a review and synthesis of the SCI and employment research becomes increasingly important not only to consolidate the current evidence but to also 
inform SCI-based vocational services and research. The current study therefore undertook a quantitative review of the published SCI research in order to examine differences in psychological functioning between employed and unemployed groups. It was broadly hypothesised that psychological variables would be positively associated with successful employment, although the magnitude of this relationship could not be determined based on the available heterogenous data.

\section{MATERIALS AND METHODS}

\section{Literature search procedure}

A search of seven electronic databases (CINAHL; Cochrane; Embase; PsycINFO; PubMed, Scopus and Web of Science) was undertaken to source eligible articles that examined psychological functioning in employed and unemployed adults with SCI. The search period dated from January 1967 (when the MeSH term 'Rehabilitation, Vocational' was introduced) to December 2013 and utilised a combination of key terms such as 'employment', 'return-to-work', 'work reintegration' and 'job re-entry' (see online Supplementary Material). Search terms were confirmed by a research librarian for accuracy. The database searches were conducted by the first author (MK) with retrieved studies then examined by both authors (MK, DD) in accordance with meta-analysis guidelines. ${ }^{19}$ An examination of available reviews ${ }^{4,5,10,11,18}$ in addition to the reference list of included studies was also undertaken by the first author (MK) to identify any further articles that may have been missed in the initial database search. ${ }^{19}$

\section{Selection criteria}

Eligible studies had to be published in a journal and in English and target an adult sample ( $\geqslant 18$ years) diagnosed with a traumatic or non-traumatic SCI. This included participants with SCI acquired in adulthood and persons with current age above 18 years (that is, possibly participants with SCI acquired in childhood). Cross-sectional, cohort and case-control designs, all of which are typical of observational studies in epidemiology, were eligible for inclusion. ${ }^{19}$ Outcome measurement was another criterion, with studies needing to evaluate a psychological factor or attribute related to adjustment outcomes following SCI. ${ }^{20}$ This included broad psychological-personal outcomes (for example, life satisfaction, quality of life, adjustment); outcomes related to internal reactions or affect (for example, thoughts, feelings, beliefs, motives), and assessment of behaviour pattern (for example, coping styles, personality). ${ }^{20}$ To increase reliability and validity, outcome measures had to be standardised. ${ }^{21}$ Moreover, studies had to provide separate data for employed and unemployed persons to allow for calculation of group differences using Cohen's $d$ effect sizes (that is, means, s.d. values, $t$-tests, one-way $F$-statistics, odds ratio, point bi-serial correlation coefficient). ${ }^{21,22}$ Finally, employment outcome had to be reported at a minimum of 1 year postinjury, as this is related to greater consistency in the results of studies. ${ }^{5}$ Employment was operationalised in line with the International Labour Organisation $^{23}$ definition, with employed individuals including paid fulltime, part-time or casual work. Unemployed individuals therefore included 'productive unemployed' (for example, volunteers) and 'non-productive' unemployed. ${ }^{23}$ Exclusion criteria included qualitative or descriptive studies, and samples that comprised individuals with co-morbid medical conditions that can negatively impact on employment outcome, namely severe brain injury or stroke. ${ }^{24,25}$

Applying the inclusion criteria to keywords, titles and abstracts resulted in an initial 783 studies. The full-text versions of these articles were subsequently retrieved and the inclusion criteria re-applied to them, resulting in 16 studies being selected (Figure 1). Notably, there were 68 studies that met all inclusion criteria (that is, for population, study design and variables) but did not specifically examine the statistical comparability of employed and unemployed individuals with SCI (see online Supplementary Material). In addition three studies used overlapping samples from the National Spinal Cord Injury Database. ${ }^{1,26,27}$ These studies were therefore combined and treated as one study to ensure independence of the data. ${ }^{21}$ This resulted in a final sample of 14 studies (Figure 1).

\section{Data collection and preparation}

Consistent with recommendations for reporting meta-analyses involving observational studies, ${ }^{19}$ a data extraction sheet was developed to summarise the key information from each study. This included data relating to the following: study characteristics (for example, sample size, recruitment source); population characteristics (for example, nationality of study participants, gender, marital status, employment status at time of injury, time since injury); and psychological measure. ${ }^{19}$ For ease of data interpretation, outcome measures were broadly categorised according to the key psychological domain they assessed: life satisfaction, quality of life, adjustment, feelings (for example, depression, anxiety), thoughts and beliefs (for example, locus of control, self-efficacy, attitudes), and behaviour pattern (for example, coping, personality traits, problem solving abilities). ${ }^{20}$ Data extraction was undertaken by the first author (MK) in consultation with the second author (DD).

\section{Statistical analysis}

Cohen's $d$ effect sizes $^{28}$ were used to determine the direction and magnitude of difference in psychological factors between employed and unemployed groups. The guidelines proposed by $\mathrm{Cohen}^{28}$ were used to interpret $d$ with $0.2,0.5$ and 0.8 equating to small, medium and large effects, respectively.

Effect sizes were computed in a multi-stage process. First, effect size estimates for each individual measure utilised by a single study were calculated. Independence of the data was ensured by utilising either the subscale or the total scores of an outcome measure to calculate $d$, but not both. ${ }^{21}$ Each effect size was subsequently sorted and examined according to the psychological domain it assessed (that is, life satisfaction, quality of life, feelings, thoughts and beliefs, and behaviour). Second, effect sizes associated with different studies that utilised the same outcome measure and subscale were pooled and averaged. Before doing so, each effect size was weighted by the study's sample size $\left(d_{\mathrm{w}}\right)$ in recognition that $N$ can have an impact on $d .^{21,22}$ Third, the effect sizes that comprised a psychological domain were aggregated and a mean effect size obtained. In the event that a study provided multiple effect sizes for a domain (that is, multiple outcome measures) these were averaged so that each study contributed only a single effect size to the average $d$ for that domain. ${ }^{21}$ The overall $d_{w}$ per domain was also weighted by the sample size of the respective studies before being averaged. ${ }^{21}$ Finally, effect sizes were standardised so that a positive $d$ indicated that the employed group, on average, performed better on the assessed psychological variable in comparison to unemployed peers.

Additional statistics were utilised to determine the statistical significance of individual and weighted effect sizes. This included ninety-five per cent confidence intervals (95\% CIs), percentage overlap scores (\% OL) and fail-safe $N s\left(N_{\mathrm{fs}}\right)$. CIs are considered to be a range of plausible values for the population mean calculated from the sample data, with statistical significance of an effect size indicated when the CI does not span zero. ${ }^{29}$ OL scores represent the extent of overlap between the employed and unemployed samples on the test measure, with smaller OL scores representing measures that are reliably sensitive to the impact of employment post-SCI. ${ }^{22}$ The calculation of $N_{\mathrm{fs}}$ statistics addresses a key criticism of meta-analysis: that of publication bias. ${ }^{30}$ The $N_{\mathrm{fs}}$ estimates the hypothetical number of additional studies required to overturn the obtained $d$ to a meaningless effect size (that is, small effect or $d<0.20$ ), whereby a larger $N_{\mathrm{fs}}$ value suggests more confidence in the findings. ${ }^{30,31}$ Given the small number of studies contributing to this meta-analysis, a conservative $N$ value was adopted, with $N_{\mathrm{fs}}$ considered adequate if it exceeded the number of studies that comprised each psychological domain (that is, $N_{\mathrm{fs}}>N$ studies per domain). ${ }^{31}$

A measure of heterogeneity $\left(I^{2}\right)$ based on the random effects model ${ }^{32,33}$ was calculated in order to describe the methodological inconsistency across studies grouped by psychological domain. Heterogeneity could only be determined for those psychological domains that comprised more than two studies. ${ }^{33}$ The value of $I^{2}$ is expressed as a percentage of between-study heterogeneity with values of $<40 \%$ being non-significant and $50-100 \%$ indicating substantial or considerable heterogeneity. ${ }^{32}$ Observed values need to be considered in 


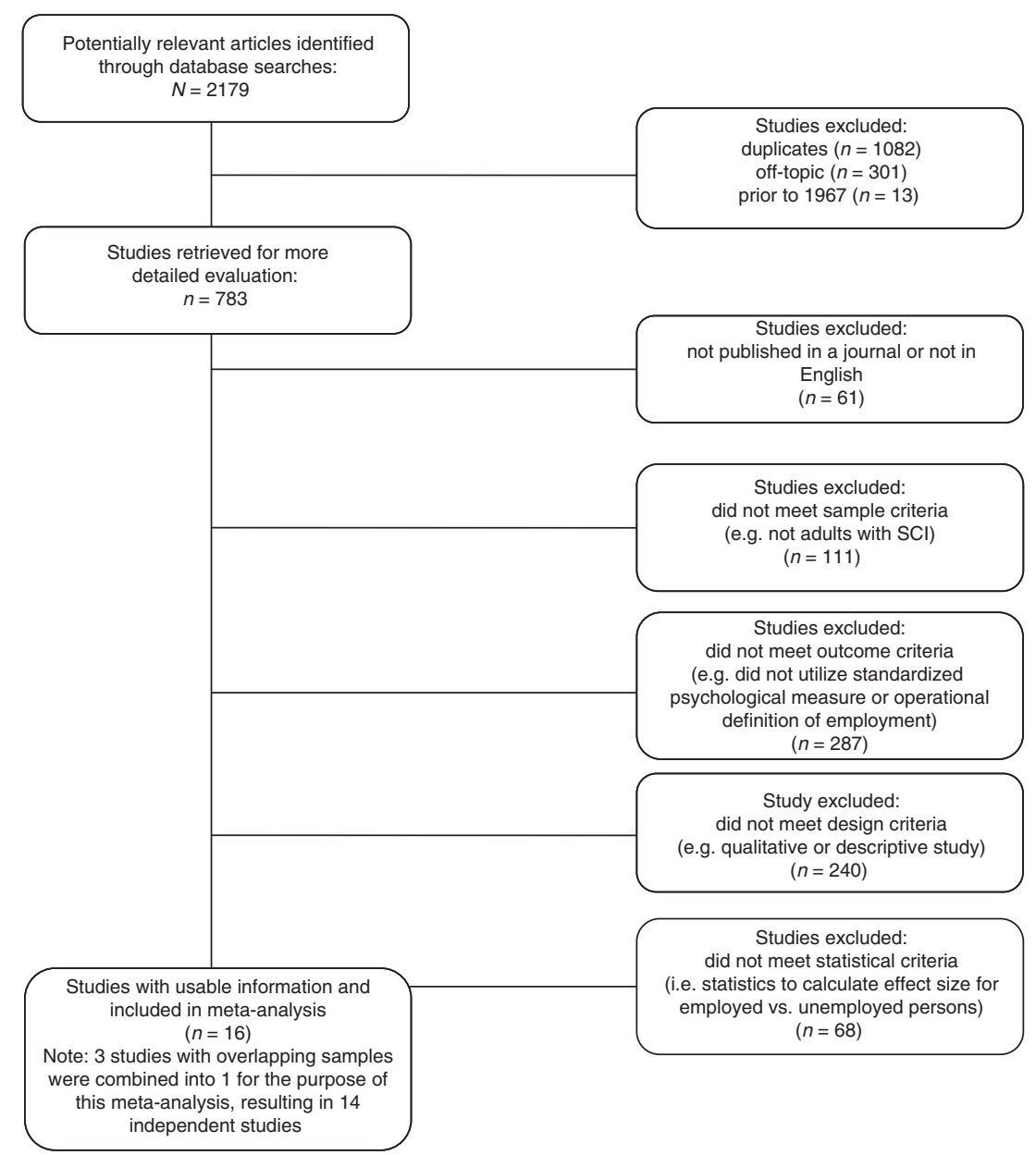

Figure 1 Flow chart of study selection.

the context of the magnitude and direction of effects and the strength of evidence for heterogeneity (that is, the CI for $I^{2 ; 32}$ ). Heterogeneity was determined using Meta-analysis with Interactive eXplanations (MIX) (version 2.0.1.4)..$^{34}$

Finally, sample size, employment status and gender were analysed as potential moderator variables. This was done by calculating Pearson correlations to assess the relationships between these moderator variables and each study's mean effect size. Employment status was defined as the number of employed persons per study. Gender was defined as the percentage of males and females in each study. These calculations were conducted using Statistical Package for the Social Sciences software (SPSS Version 20. 0.0.1, IBM, Armonk, NY, USA).

The conclusions drawn from this meta-analysis are therefore based on the combination of these statistics. A psychological domain was considered clinically important to employment if it was associated with a medium $(d \geqslant 0.50)$ to large $d(d \geqslant 0.80)^{28}$ that was statistically significant (that is, $95 \%$ CIs did not span zero), and an $N_{\mathrm{fs}}$ score that was sufficient to suggest that the findings were unlikely to be compromised by the file drawer problem (that is, $N_{\mathrm{fs}}>N$ studies per domain). The interpretation of these findings was undertaken in the context of study heterogeneity.

\section{RESULTS}

\section{Study characteristics}

The 14 independent studies ${ }^{1,12,14,15,26,27,35-44}$ included in the meta-analysis provided data for 9868 participants (Table 1). The majority of the data originated from the USA ( $n=10$ studies), with studies from Australia ( $n=2$ studies), Canada ( $n=1$ study) and Norway ( $n=1$ study) also contributing. Sample sizes ranged from small-scale to multi-centre trials, with two studies (that by Krause and Broderick, ${ }^{15}$ and the combined study of Hess et al., ${ }^{1}$ Dowler et al. ${ }^{26}$ and Meade et al. ${ }^{27}$ ) contributing to $74 \%$ of the overall sample size. Study recruitment was selective, with all participants sourced from health services, although a few studies additionally recruited from SCI associations ( $n=2$ studies); public advertising ( $n=2$ studies); an employment service provider $(n=1$ study); and through a university ( $n=1$ study). All studies involved survey-based, cross-sectional designs undertaken via interview $(n=8$ studies) and/or postal mail ( $n=6$ studies), or by retrospective review of data ( $n=2$ studies).

In total, 14 standardised psychological measures were utilised across the 14 studies. All were self-report surveys, with one study $^{43}$ specifying that assessments were undertaken in conjunction with a clinician. The most commonly used and validated SCI measure was the Satisfaction with Life Scale ( $n=4$ studies), with the remaining outcome measures utilised by only one or two studies.

In relation to effect size estimation, nine studies provided means, s.d. or odds ratios from which to calculate $d$. The remaining four studies ${ }^{15,36,41,42}$ required some estimation (for example, provided $t$-test values) in the calculation of $d$. As such, there was a relatively high consistency in the computation of effect sizes. ${ }^{21}$ 
Table 1 Descriptive characteristics of participants $(N=14$ studies $)$

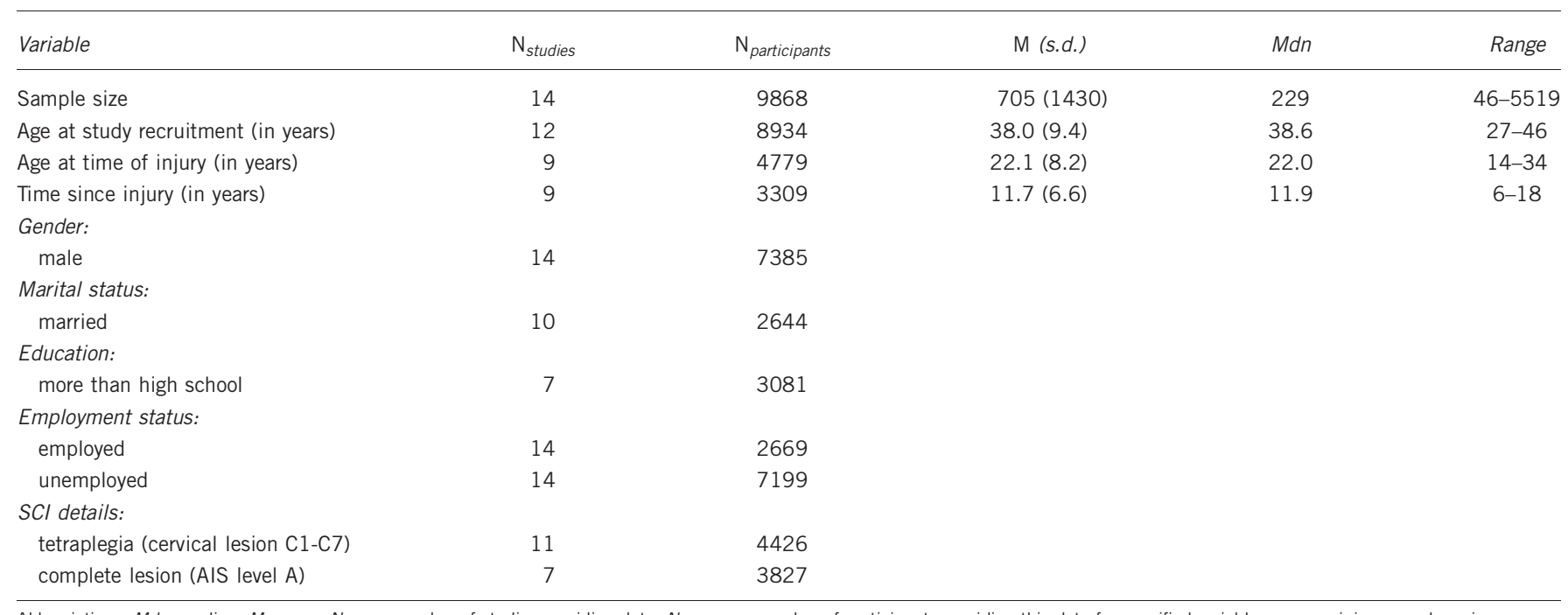

Abbreviations: $M d n$, median; $M$, mean; $N_{\text {studies }}$, number of studies providing data; $N_{\text {participants, }}$ number of participants providing this data for specified variable; range, minimum and maximum average value across the studies reporting this data; s.d., standard deviation.

\section{Participant characteristics}

Combined demographic details of all participants are shown in Table 1. Details of SCI diagnosis, in accordance with the International Standards for Neurological Classification, were not consistently reported across the studies. The average employment rate (for fulltime or part-time employment, as defined by the ILO) was $27 \%$, although this varied from $14 \%{ }^{27}$ to $64 \% .{ }^{35}$ Employment status at the time of injury was not consistently reported, with only two studies reporting rates ranging from 78 to $86 \% .^{1,43}$

\section{Psychological variables associated with employment}

The weighted mean effect sizes for each psychological domain, rank ordered by size, are listed in Table 2. Based on the study criteria for significance (that is, $d \geqslant 0.50 ; 95 \%$ CIs did not span zero; $N_{\mathrm{fs}}>N$ studies per domain), three domains were associated with weighted $d s$ that could be considered clinically important to employment: feelings, quality of life and life satisfaction.

The low $\%$ OL and high $N_{\mathrm{fs}}$ statistics associated with these domains also suggests that these may be areas that can differentiate between those who are employed and those who are unemployed. For example, $94.2 \%$ of employed people reported scores across individual measures of depression (Brief Patient Health Questionnaire, Centre for Epidemiological Studies Depression Scale) that were not obtained by the unemployed group. However, these results also need to be considered in the context of considerable between-study heterogeneity $\left(I^{2}>75 \%\right.$; Table 3$)$. In comparison, statistical homogeneity was noted for the psychological domain of life satisfaction, with associated positive effect sizes ranging from 0.37 to 0.85 .

In relation to the thoughts and beliefs domain, individual measures of vocational attitude (My Vocational Situation Scale) and self-efficacy (Moorong Self-efficacy Scale) were associated with positive and moderate effects $\left(d_{\mathrm{w}}>0.40\right)$, albeit small $N_{\mathrm{fs}}$ values, whereas unemployed and employed persons obtained similar scores on the Multidimensional Locus of Control Scale. Adjustment to SCI, as measured by the Multidimensional Adjustment Profile $(d=0.40)$, in addition to patterns of experience and behaviour, as determined by standardised personality inventories (Multidimensional Personality QuestionnaireMPQ; Zuckerman-Kuhlman Personality Questionnaire-ZKPQ) were only weakly associated with employment.

\section{Moderator analyses}

The correlation between sample size and mean weighted effect size ( $r=0.42, \quad P=0.16$ ) was not statistically significant (two-tailed). Similarly, the ratio of employed persons across the 14 studies did not have an impact on $d(r=0.40, P=0.15)$. In comparison, higher $d$ 's were associated with studies that comprised a greater proportion of males $(r=0.56, P=0.04)$. However, this significant result needs to be interpreted with caution due to the likelihood of a Type I error (that is, false-positive association) resulting from the relatively small number of mean effect sizes $(n=14)$ contributing to this finding.

\section{DISCUSSION}

The current study used meta-analytic techniques to consolidate and evaluate 14 studies examining the psychological characteristics of employed and unemployed peers with SCI. Individual measures of affective experience or feelings, in addition to quality of life and life satisfaction demonstrated more than negligible associations with employment. However, when interpreting the findings in the context of publication bias and study heterogeneity, the most compelling finding was the medium to large effect size associated with life satisfaction, with employed persons reporting greater endorsement in this area than unemployed peers. Effect sizes associated with thoughts and beliefs, adjustment and behaviour pattern were largely nonsignificant. This indicates that more information is required in order to establish the psychological correlates of employment.

These findings, although preliminary, are consistent with current literature reporting that psychological resources such as affective coping and perceived satisfaction ${ }^{11,17}$ are significantly related to vocational activity post SCI. There were also discrepancies between this study and the available SCI literature. In particular, locus of control did not significantly differentiate employed from unemployed individuals. ${ }^{15}$ It may be that an internal locus of control can both impede and enhance psychological well-being following SCI. ${ }^{45,46}$ In addition, individual personality traits such as achievement, aggression, sociability and stress reaction (as measured by the MPQ and ZPQ in this meta-analysis) were weakly associated with employment. However, this latter finding may also be explained by the fact that personality traits are conceptually defined as stable characteristics, hence less likely to be affected by employment. ${ }^{20}$ 
Table 2 Cohen's $d$ effect sizes tests for psychological measures administered to adults with $\mathrm{SCl}$ who were employed or unemployed

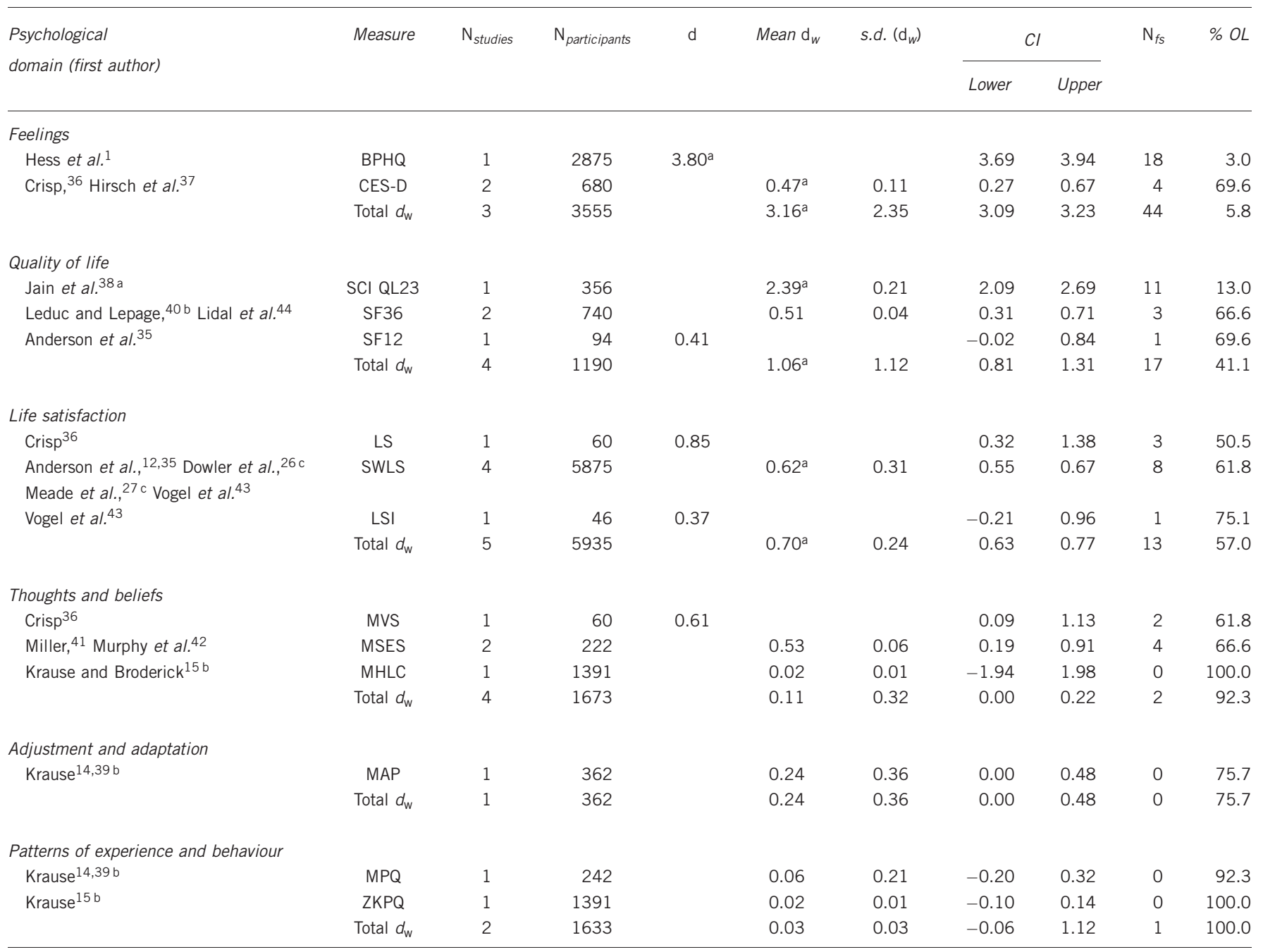

Abbreviations: BPHQ, Brief Version of the Patient Health Questionnaire; CES-D, Center for Epidemiologic Studies Depression Scale; Cl, $95 \%$ confidence intervals; $d$, Cohen's $d ; d_{w}$, weighted mean effect size calculated for domain totals (note: weighting only applied when effect sizes were calculated using data from two or more studies); LS, Life Satisfaction Scale; LSI, Life Satisfaction Index; MAP, Multidimensional Adjustment Profile; MHLC, Multidimensional Health Locus of Control Scale; MPQ, Multidimensional Personality Questionnaire; MSES, Moorong Self-efficacy Scale;

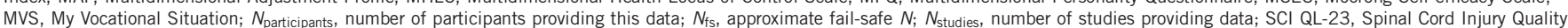
of Life Questionnaire; s.d. $\left(d_{w}\right)$, standard deviation of the effect size; SF-12, Short-form Health Survey (12-item); SF-36, Short-form Health Survey (36-item); SWLS, Satisfaction With Life Scale; of Life Questionnaire; s.d. $\left(d_{w}\right)$, standard deviation of the effect size; SF-12, Short-form Health Survey (12-item); SF-36, Short-form
ZKPQ, Zuckerman-Kuhlman Personality Questionnaire; \% OL, percentage overlap scores between employed and unemployed groups.

${ }^{a}$ Effect size met the study criteria: $d \geqslant 0.50 ; 95 \% \mathrm{Cls}$ did not span zero; $N_{\mathrm{fs}}>N$ studies per domain. Note: a positive $d$ value indicates that the employed group, on average, performed better on the assessed psychological variable in comparison to unemployed peers.

${ }^{b}$ Average effect size calculated for study, based on several subscales originating from the same measure.

cStudies combined due to overlapping samples.

The current findings have a number of important implications for vocational rehabilitation practice in SCI rehabilitation. In particular the significant association between reduced symptoms of depression and successful work outcome reported by Hess et al., ${ }^{1}$ among individuals at an average of 10 years $($ s.d. $=8.17)$ post SCI, highlights the importance of undertaking targeted psychological assessment and intervention during the early stages of rehabilitation in order to relieve emotional distress and, potentially, maximise employment outcome. ${ }^{4,5,8,9,47}$ There is also the distinct possibility that employment has a significant impact on $\operatorname{mood}{ }^{8}$ Consequently, rehabilitation interventions to preserve employment and facilitate job finding may help to establish an early expectation of a continuation of work or entry into the job market, in addition to optimising psychological outcome. ${ }^{5,48}$ These interventions need to be collaborative, with the clinician working in partnership with the individual, health-care providers and labour services in order to maximise vocational potential. ${ }^{5,48}$ However, consideration also needs to be given to the period of time between an individual's discharge from the in-patient spinal injury rehabilitation unit and employment. ${ }^{6}$ For some people, the psychological impact of a SCI is chronic, hence there may be a considerable delay before employment goals are achieved. . $^{3,6,8,49}$ There is a need then, for future research to examine referral pathways and, in particular, to explore barriers to the use of vocational services for people with SCI.

\section{Study limitations}

There are a number of limitations that warrant consideration. First, missing data is an issue, as not all studies may have been captured by the search criteria. In recognition of this limitation, attempts were made to ensure a comprehensive database search, which included investigation of reference lists of the included studies and available 
Table 3 Heterogeneity by psychological domain ${ }^{\mathrm{a}}$

\begin{tabular}{lccrrrr}
\hline \multirow{2}{*}{ Domain } & $\mathrm{N}_{\text {studies }}$ & $\mathrm{N}_{\text {participants }}$ & $\mathrm{I}^{2}$ & \multicolumn{2}{c}{$\mathrm{Cl}$} \\
\cline { 4 - 6 } & & & & & Lower & Upper \\
\hline Feelings & 3 & 3555 & 99.87 & 99.83 & 99.90 \\
Quality of life & 4 & 1190 & 98.47 & 97.30 & 99.13 \\
Life satisfaction & 5 & 5935 & 32.38 & 0.00 & 85.94 \\
Thoughts and beliefs & 4 & 1673 & 79.45 & 34.63 & 93.54 \\
\hline
\end{tabular}

Abbreviations: $\mathrm{Cl}, 95 \%$ confidence intervals; $P$, heterogeneity index; $N_{\text {studies }}$, number of studies providing data; $N_{\text {participants }}$, number of participants providing this data.

aHeterogeneity index only calculated for domains that comprised more than two studies. ${ }^{33}$

reviews. Fail-safe $N$ statistics were additionally calculated in order to address the possibility that there may have been unpublished studies that failed to find effects in line with this review's aims and/or studies that addressed employment outcome post-SCI. However, $N_{\mathrm{fs}}$ statistics do not completely alleviate the problem of publication bias. ${ }^{30,31}$

Second, the identified studies involved cross-sectional self-report surveys, with only one study ${ }^{43}$ incorporating psychological ratings by significant others or by clinicians. This highlights the subjective nature of the available SCI research. Indeed, a particular limitation here is that people may conceive of abstract psychological constructs in different ways depending on their context, arising through individual differences in their health condition as well as environmental and personal factors. ${ }^{20}$ The criterion to only include data from standardised psychological measures partly addressed this limitation.

Third, the sample pool was highly selective, with the majority of studies stemming from the United States and North America in addition to recruiting participants from public health services. In saying this, generalisation of the current results to different countries is also challenging given the diversity in national employment and unemployment rates, vocational rehabilitation practices and funding systems. ${ }^{4,5}$

Fourth, included studies were either cross-sectional or did not assess key psychological variables at both the time of employment and then at the time of unemployment. Longitudinal studies are therefore needed to clarify the direction of the relationship between employment and the psychological factors and attributes assessed in this meta-analysis. Indeed, research indicates that broad outcomes of psychological adjustment to SCI, such as life satisfaction and adjustment, can significantly change over time in response to stressors including the stress of job loss. ${ }^{48,50}$

Finally, the included studies did not consistently report sociodemographic or injury variables, thereby limiting potential moderator analyses. For instance, although pre-injury employment has been identified as an important factor related to employment, ${ }^{4}$ this was only reported by two studies. ${ }^{1,43}$ In addition, details on the occupational positions obtained, pre- and post SCI, would help to identify the type of vocational retraining required during rehabilitation, including opportunities for higher education. ${ }^{4}$ Time since injury is another critical variable for employment, ${ }^{6}$ yet few studies reported this detail. Additional sub-group analyses might include the differential effects of employment (that is, part-time vs full-time employment) on the examined psychological indices, ${ }^{51}$ and the impact of gender on vocational interests and engaged work roles. ${ }^{4,52}$ Another contributing factor relates to patient characteristics, namely diversity in level of injury and associated medical comorbidities (for example, chronic neuropathic pain ${ }^{49,51}$ ). SCI research indicates that high cervical injuries with complex comorbidities may not be compatible with work or require specialised, intense assistance for vocational rehabilitation to be successful. ${ }^{48}$ In combination, these limitations contribute to a lessening of the clarity of findings within this meta-analysis and suggest that the results cannot be generalised beyond the examined population.

Notwithstanding these limitations, the current study helps to inform the evidence-base for clinical practice in SCI vocational rehabilitation, by consolidating the available research and drawing attention to the nature of interventions that are likely to be supportive in respect to facilitating employment. Further large-scale and longitudinal research focussing on the assessment of psychological contributors to, or effects of, work retention in this population will help to confirm these findings.

\section{DATA ARCHIVING}

There were no data to deposit.

\section{CONFLICT OF INTEREST}

The authors declare no conflict of interest.

\section{ACKNOWLEDGEMENTS}

We are very grateful to M Bell, Research Librarian, University of Adelaide, for assistance with the electronic database searches.

1 Hess DW, Meade MA, Forchheimer M, Tate DG. Psychological well-being and intensity of employment in individuals with a spinal cord injury. Top Spinal Cord Inj Rehabil 2004; 9: 1-10.

2 Kuipers P, Kendall MB, Amsters D, Pershouse K, Schuurs S. Descriptions of community by people with spinal cord injuries: concepts to inform community integration and community rehabilitation. Int J Rehabil Res 2011; 34: 167-174.

3 Murphy G, Middleton J, Quirk R, De Wolf A, Cameron ID. Prediction of employment status one year post-discharge from rehabilitation following traumatic spinal cord injury: an exploratory analysis of participation and environmental variables. J Rehabil Med 2009; 41: 1074-1079.

4 Ottomanelli L, Lind L. Review of critical factors related to employment after spinal cord injury: Implications for research and vocational services. J Spinal Cord Med 2009; 32: 503-531.

5 Young AE, Murphy GC. Employment status after spinal cord injury (1992-2005): a review with implications for interpretation, evaluation, further research, and clinical practice. Int J Rehabil Res 2009; 32: 1-11.

6 Ramakrishnan K, Mazlan M, Julia PE, Abdul Latif L. Return to work after spinal cord injury: factors related to time to first job. Spinal Cord 2011; 49: 924-927.

7 Gobelet C, Luthi F, Al-Khodairy AT, Chamberlain MA. Vocational rehabilitation: a multidisciplinary intervention. Disabil Rehabil 2007; 29: 1405-1410.

8 Murphy G, Young AE, Brown DJ, King NJ. Explaining labor force status following spinal cord injury: the contribution of psychological variables. J Rehabil Med 2003; 35: 276-283.

9 Chapin MH, Kewman DG. Factors affecting employment following spinal cord injury: a qualitative study. Rehabil Psychol 2001; 46: 400-416.

10 Whalley Hammell K. Quality of life after spinal cord injury: a meta-synthesis of qualitative findings. Spinal Cord 2007; 45: 124-139.

11 Dijkers M. Quality of life after spinal cord injury: a meta analysis of the effects of disablement components. Spinal Cord 1997; 35: 829-840.

12 Anderson CJ, Krajci KA, Vogel LC. Life satisfaction in adults with pediatric-onset spinal cord injuries. J Spinal Cord Med 2002; 25: 184-190.

13 Geyh S, Nick E, Stirnimann D, Ehrat S, Michel F, Peter C et al. Self-efficacy and selfesteem as predictors of participation in spinal cord injury: an ICF-based study. Spinal Cord 2012; 50: 699-706.

14 Krause JS. Personality and traumatic spinal cord injury: Relationship to participation in productive activities. J App/ Rehabil Couns 1997; 28: 15-20.

15 Krause JS, Broderick L. Relationship of personality and locus of control with employment outcomes among participants with spinal cord injury. Rehabil Couns Bull 2006; 49: 111-114.

16 Murphy G, Young AE. Employment participation following spinal cord injury: relation to selected participant demographic, injury and psychological characteristics. Disabil Rehabil 2005; 27: 1297-1306.

17 Burns SM, Boyd BL, Hill J, Hough S. Psychosocial predictors of employment status among men living with spinal cord injury. Rehabil Psychol 2010; 55: 81-90. 
18 Ullrich PM, Spungen AM, Atkinson D, Bombardier CH, Yuying C, Erosa NA et al. Activity and participation after spinal cord injury: state-of-the-art report. J Rehabil Res Dev 2012; 49: 155-174.

19 Stroup DF, Berlin JA, Morton SC, Olkin I, Williamson GD, Rennie D et al. Meta analysis of observational studies in epidemiology: a proposal for reporting. Meta-analysis of observational studies in epidemiology (MOOSE) group. JAMA 2000; 283: 2008-2012.

20 Geyh S, Muller R, Peter C, Bickenbach JE, Post MWM, Stucki G et al. Capturing the psychologic-personal perspective in spinal cord injury. Am J Phys Med Rehabil 2011; 90(suppl), S79-S96.

21 Lipsey MW, Wilson DB. Practical Meta-analysis. Sage Publications: London, UK, 2001

22 Zakzanis KK. Statistics to tell the truth, the whole truth, and nothing but the truth: formulae, illustrative numerical examples, and heuristic interpretation of effect size analyses for neuropsychological researchers. Arch Clin Neuropsych 2001; 16 : 653-667

23 International Labour Organization. Resolution concerning statistics of the economically active population, employment, unemployment and underemployment, adopted by the Thirteenth International Conference of Labour Statisticians, Geneva, 1982. Available at http://www.ilo.org 23.

24 Shames J, Treger I, Ring H, Giaquinto S. Return to work following traumatic brain injury: trends and challenges. Disabil Rehabil 2007; 29: 1387-1395.

25 Wolfenden B, Grace M. Returning to work after stroke: a review. Int J Rehabil Res 2009; 32: 93-97.

26 Dowler R, Richards JS, Putzke JD, Gordon W, Tate D. Impact of demographic and medical factors on satisfaction with life after spinal cord injury: A normative study. J Spinal Cord Med 2001; 24: 87-91.

27 Meade M A, Lewis A, Jackson MN, Hess DW. Race, employment, and spinal cord injury. Arch Phys Med Rehab 2004; 85: 1782-1792.

28 Cohen J. A power primer. Psychol Bulletin 1992; 112: 155-159.

29 Cumming G, Finch S. Inference by eye. Confidence intervals and how to read pictures of data. Am Psychol 2005; 60: 170-180.

30 Rosenthal R. The 'file drawer problem' and tolerance for null results. Psychol Bult 1979; 86: 638-641.

31 Orwin RG. A fail-safe N for effect size in meta-analysis. J Educat Stat 1983; 8: 157-159.

32 Higgins JP and Green S (Eds.) Cochrane Handbook for Systematic Reviews of Interventions Version 5.1.0. The Cochrane Collaboration: London, UK, 2011, Available at www.handbook-cochrane.org.

33 Valentine J C, Pigott TD, Rothstein HR. How many studies do you need? A primer on statistical power for meta-analysis. J Educat Behav Stat 2010; 35: 215-247.

34 Bax L. (2011). MIX 2.0_professional software for meta-analysis in Excel. Version 2.0.1.4. BiostatXL. Available at http://www.meta-analysis-made-easy.com.

35 Anderson CJ, Vogel LC, Willis KM, Betz RR. Stability of transition to adulthood among individuals with pediatric-onset spinal cord injuries. J Spinal Cord Med 2006; 29: $46-56$.
36 Crisp R. Vocational decision making by sixty spinal cord injury patients. Paraplegia 1992; 30: 420-424.

37 Hirsch AT, Molton IR, Johnson $\mathrm{KL}$, Bombardier $\mathrm{CH}$, Jensen MP. The relationship of chronological age, age at injury, and duration of injury to employment status in individuals with spinal cord injury. Psychol Inj Law 2009; 2: 263-275.

38 Jain NB, Sullivan M, Kazis LE, Tun CG, Garshick E. Factors associated with healthrelated quality of life in chronic spinal cord injury. Am J Phys Med Rehabil 2007; 86: 387-396.

39 Krause JS, Anson CA. Adjustment after spinal cord injury: relationship to participation in employment or educational activities. Rehabil Couns Bull 1997; 40: 202-214.

40 Leduc BE, Lepage Y. Health-related quality of life after spinal cord injury. Disabil Rehabil 2002; 24: 196-202.

41 Miller SM. The measurement of self-efficacy in persons with spinal cord injury: psychometric validation of the moorong self-efficacy scale. Disabil Rehabil 2009; 31: 988-993.

42 Murphy G, Middleton J, Quirk R, De Wolf A, Cameron ID. Predicting employment status at 2 years' postdischarge from spinal cord injury rehabilitation. Rehabil Psychol 2011; 56: 251-256.

43 Vogel LC, Klaas SJ, Lubicky JP, Anderson CJ. Long-term outcomes and life satisfaction of adults who had pediatric spinal cord injuries. Arch Phys Med Rehab 1998; 79. 1496-1503.

44 Lidal IB, Veenstra M, Hjeltnes N, Biering-Sørensen F. Health-related quality of life in persons with long-standing spinal cord injury. Spinal Cord 2008; 46: 710-715.

45 Craig A, Franklin J, Andrews G. A scale to measure locus of control of behaviour. $\mathrm{Br} J$ Med Psychol 1984; 57: 173-180.

46 Chung MC, Preveza E, Papandreou K, Prevexas N. The relationship between posttraumatic stress disorder following spinal cord injury and locus of control. J Affect Disord 2006; 93: 229-232.

47 Bonanno GA, Kennedy P, Galatzer-Levy IR, Lude P, Elfström ML. Trajectories of resilience, depression, and anxiety following spinal cord injury. Rehabil Psychol 2012; 57: 236-247.

48 Wegener ST, Adams LL, Rohe D. Promoting optimal functioning in spinal cord injury: the role of rehabilitation psychology. Handb Clin Neurol 2012; 109. 297-314.

49 Ullrich PM, Lincoln RK, Tackett MJ, Miskevics S, Smith BM, Weaver FM. Pain depression, and health care utilization over time after spinal cord injury. Rehabil Psychol 2013; 58: 158-165.

50 Krause JS. Employment after spinal cord injury: transition and life adjustment. Rehabil Couns Bull 1996; 39: 244-255.

51 Westgren N, Levi R. Quality of life and traumatic spinal cord injury. Arch Phys Med Rehabil 1998; 79: 1433-1439.

52 Krause JS, Saunders LL, Staten D, Rohe DE. Vocational interests after recent spinal cord injury: comparisons related to sex and race. Arch Phys Med Rehabil 2011; 92 626-631.

Supplementary Information accompanies this paper on the Spinal Cord website (http://www.nature.com/sc) 